\title{
Radial artery vs saphenous vein graft used as the second conduit for surgical myocardial revascularization: long-term clinical follow-up
}

Ivana Petrovic, Dusko Nezic, Miodrag Peric, Predrag Milojevic, Olivera Djokic, Dragana Kosevic, Nebojsa Tasic, Bosko Djukanovic and Petar Otasevic

\begin{abstract}
Background: There is ongoing debate regarding the efficacy of the radial artery (RA) as an aortocoronary conduit, with few solid data regarding long-term clinical results. We sought to determine if the use of the RA as the second arterial conduit, beside left internal thoracic artery (LITA), would improve long-term clinical outcome after CABG as compared to saphenous vein graft (SVG).

Methods: Between March 2001 and November 2003, 200 patients underwent isolated CABG and were randomized in 1:1 fashion to receive either LITA and RA grafts or LITA and SVGs. The primary end point was composite of cardiovascular mortality, non-fatal myocardial infarction and need for repeat myocardial revascularization (either surgical or percutaneous).

Results: There was no significant difference in absolute survival, with 12 deaths in each group during the study period (log rank $=0.01, p=0.979$ ). There were 3 and 2 cardiac deaths in RA and SVG groups, respectively. There was no difference in long-term clinical outcome between the groups (log rank $=0.450, p=0.509$ ). Eleven patients in RA group had one or more non-fatal events; 7 patients suffered a myocardial infarction, 9 patients underwent percutaneous coronary angioplasty, and 1 patient required redo coronary surgery. Likewise, 13 patients in SVG group had non-fatal event; 7 patients had myocardial infarction, 13 patients had percutaneous coronary intervention and 3 patients required redo coronary surgery. Angiograms were performed in 23 patients in RA group (patency rate 92 \%) and 24 in SVG group (patency rate $86 \%)(p=0.67)$.

Conclusion: In this small randomised study our data indicate that there is no difference in the 8 year clinical outcomes in relatively young patients between those having a RA or a saphenous vein graft used as a second conduit, beside LITA, for surgical myocardial revascularisation.
\end{abstract}

Keywords: Radial artery graft, Saphenous vein graft, Clinical outcome

\section{Background}

Left anterior descending (LAD) coronary artery bypass grafting (CABG) with the left internal thoracic artery (LITA) is regarded as the gold standard in coronary bypass surgery [1]. Initially described in 1973 by Carpantier and colleagues [2] the radial artery (RA) was soon abandoned as a bypass graft as reports documented dismal early angiographic outcomes [2, 3]. However, with various methods to counter spasms, such as a block harvesting,

\footnotetext{
* Correspondence: potasevic@yahoo.com

Dedinje Cardiovascular Institute and Belgrade University School of Medicine, Belgrade, Serbia
}

(c) 2015 Petrovic et al. Open Access This article is distributed under the terms of the Creative Commons Attribution 4.0 International License (http://creativecommons.org/licenses/by/4.0/), which permits unrestricted use, distribution, and reproduction in any medium, provided you give appropriate credit to the original author(s) and the source, provide a link to the Creative Commons license, and indicate if changes were made. The Creative Commons Public Domain Dedication waiver (http://creativecommons.org/publicdomain/zero/1.0/) applies to the data made available in this article, unless otherwise stated.

the long-term outcome has improved significantly, and the RA is currently being used as the second graft of choice after the LITA in many institutions [3, 4].

Most literature reports [5-10] demonstrate excellent patency rates in protocol-driven studies and in symptomatic patients. Reported RA patency rates range from 83 to $98 \%$ at $1-7$ years after CABG. However, although most clinical and patency reports regarding the RA have been favorable, some are not $[11,12]$. Hence ongoing doubt and debate remain regarding the efficacy of the RA as an aortocoronary conduit, with few solid data regarding the medium-term patency rate of these grafts. 
The aim of this study was to determine if the use of the RA as the second arterial conduit, beside LITA, would improve long-ter $\mathrm{m}$ clinical outcome after CABG as compared to saphenous vein graft (SVG).

\section{Methods \\ Patients}

Between March 2001 and November 2003, 200 patients underwent isolated CABG and were randomized in 1:1 fashion to receive either LITA and RA grafts or LITA and SVGs. Use of additional SVGs was permitted in both groups depending on angiographic findings. The study protocol was approved by Institutional Ethics Committee, and investigation conforms to the principles outlined in the Declaration of Helsinki. Written consent was obtained from all patients prior to the procedure.

Patients were included in the study if at least one target vessel for RA/SVG grafting had at least $80 \%$ stenosis, was at least $1.5 \mathrm{~mm}$ in diameter, and had no diffuse distal disease. Patients were excluded in the case of a single-vessel disease and if they had undergone any concomitant acquired or congenital cardiac or aortic surgery. Hemodialysis was considered a strong contraindication for RA harvesting due to a concern about the need for possible upper limb dialysis access. The exclusion criteria were a positive Allen's test, a history of Raynaud's syndrome or vasculitis. In all cases, before RA harvesting, the adequacy of ulnar compensation was assessed by the Doppler method. The RA was always harvested from the non-dominant arm; bilateral RA harvesting was never performed.

\section{Procedures}

All patients underwent conventional angiography before surgery using retrograde femoral artery catheterization under standard fluoroscopy using an iodine contrast agent. Each angiogram was evaluated by two experienced cardiologists and the decisions were made by consensus. During the follow-up period coronary angiographies were performed if clinically indicated.

Complete echocardiographic examination was performed in all patients prior to index surgery. Left ventricular ejection fraction was assessed using Simpson biplane formula.

All patients were operated at the Dedinje Cardiovascular Institute, Belgrade, Serbia, a tertiary care center. Open harvest of the RA was used in all patients. For the myocardial protection purposes we used $600-1000 \mathrm{ml}$ of cold antegrade modified St. Thomas cardioplegic solution to initially arrest the heart. Topical cooling of the heart was used during procedure (ice slash). Cardioplegia was repeated only if cross-clamp time exceeded $90 \mathrm{~min}$. Cold blood cardiopegia was used only in patients with a EF
$<30 \%$. Side-biting clamps were used for performing proximal anastomoses in all patients. All RA grafts were deployed to the artery with at least $80 \%$ stenosis, providing that it is considered an important coronary artery (smaller, same territory arteries or arteries supplying heavily infarcted areas were not grafted with radial artery). During or after the procedure no intravenous drugs were given to prevent RA spasm. However, we used topically verapamil and nitroglycerin solution (balanced to $\mathrm{pH}$ 7.4). All radial arteries were rinsed after harvesting and kept in this solution before implantation. All of our patients were given oral preparations of the calcium channel blockers during one year after surgery to prevent RA spasm.

\section{Follow-up}

Patients were followed for 8 years since index surgery for the composite of cardiovascular mortality, non-fatal myocardial infarction and need for repeat myocardial revascularization (either surgical or percutaneous). Data were collected either by phone or during visits.

\section{Statistical analysis}

The data were entered into an electronic database (Access, Microsoft) and analyzed using the SPSS 16.0 software (SPSS Inc.). Continuous variables were expressed as mean and standard deviations. Categorical variables were expressed as percentages. Dichotomous variables were analyzed using the $\chi^{2}$ test and Fisher's exact test, and continuous variables were analyzed using the $t$-test. Binary logistic regression analyses with the fixed entry method were performed in order to identify predictors for RA graft occlusion. The parameters examined were defined, and included established risk factors for coronary artery disease. Accordingly, those parameters with the lowest $p$ values in the univariate analysis were entered into the regression model; $p<0.05$ was considered statistically significant throughout.

\section{Results}

\section{Baseline data}

The baseline demographic and clinical data of the patients are depicted in Table 1. The groups were well balanced with respect to demographic, clinical and angiographic data. Briefly, patients were predominantly males, in their mid-fifties, around $40 \%$ were diabetic, and more than $50 \%$ of patients in both groups had previous myocardial infarction. Mean left ventricular ejection fraction was slightly decreased, and the majority of patients had triple vessel coronary artery disease.

\section{Operative and perioperative data}

There were no perioperative deaths in both groups. The average number of implanted grafts was similar in patients 
Table 1 Baseline characteristics of study patients

\begin{tabular}{llll}
\hline & LITA/RA/SVG & LITA/SVG & $P$ value \\
\hline Male/female ratio & $73 / 27$ & $73 / 27$ & 1.0 \\
Mean age (years) & $56.3 \pm 6.1$ & $57.1 \pm 6.5$ & 0.29 \\
Risk factors and comorbidities & & & \\
Diabetes & $39 \%$ & $43 \%$ & 0.56 \\
Smoking & $67 \%$ & $65 \%$ & 0.76 \\
Dyslipidemia & $75 \%$ & $74 \%$ & 0.87 \\
Hypertension & $92 \%$ & $89 \%$ & 0.47 \\
PAD & $12 \%$ & $14 \%$ & 0.67 \\
Previous stroke & $3 \%$ & $2 \%$ & 0.65 \\
COPD & $9 \%$ & $8 \%$ & 0.80 \\
Previous MI & $57 \%$ & $56 \%$ & 0.85 \\
Number of diseased vessels & $3.08 \pm 0.66$ & $3.14 \pm 0.66$ & 0.52 \\
Mean LVEF & $48.8 \pm 10.7 \%$ & $48.0 \pm 10.8 \%$ & 0.60 \\
Coronary artery disease: & & & \\
Left main stenosis & 26 & 24 & 0.74 \\
Double vessel disease & 17 & 16 & 0.92 \\
Triple vessel disease & 83 & 83 & 1.0 \\
\hline Abbreviations: COPD chronic obstructive pulmonary disease, LITA left internal \\
thoracic artery, MI myocardial infarctin, PAD peripheral artery disease, RA radial \\
artery, SVG saphenous vein graft
\end{tabular}

who received RA or SVG $(3.08 \pm 0.66$ vs $3.14 \pm 0.66$, respectively). All the patients in both groups had LITA grafting on LAD implanted. Table 2 details placement of RA grafts. Briefly, the majority of RA grafts were placed either on first $(50 \%)$ or on second (15\%) obtuse marginal branch. RA graft was never placed to the right coronary artery or diagonal branch if they were previously occluded. Perioperative complications are detailed in Table 3. There were no difference between the groups, with a total of 47 events in RA group and 45 events in SVG group $(p=0.89)$. Although numerous, events were mostly mild and resolved upon instution of adequate therapy. Atrial fibrillation was most frequent adverse event in both groups, followed by pleural effusion and hemorrhage. The average length of index hospitalization was 8 days.

\section{Follow-up}

All patients were followed for 8 years or until death. There was no significant difference in absolute survival,

Table 2 Distribution of radial artery graft placement

\begin{tabular}{ll}
\hline & Radial artery \\
\hline Diagonal branch & $9 / 100(9 \%)$ \\
Ramus intermedius & $9 / 100(9 \%)$ \\
First obtuse marginal branch & $50 / 100(50 \%)$ \\
Second obtuse marginal branch & $15 / 100(15 \%)$ \\
Right coronary artery & $17 / 100(17 \%)$ \\
\hline
\end{tabular}

Table 3 Adverse events after index surgery

\begin{tabular}{lllc}
\hline & LITA/RA/SVG & LITA/SVG & P value \\
\hline TIA/Stroke & 3 & 2 & 0.65 \\
Sternal dehiscence & 1 & 2 & 0.56 \\
Radial nerve exploration & 1 & 0 & 0.31 \\
Leg wound infection & 0 & 2 & 0.15 \\
Pericardial effusion & 4 & 3 & 0.70 \\
Pleural effusion & 7 & 6 & 0.77 \\
Perioperative myocardial infarction & 3 & 4 & 0.70 \\
Atrial fibrillation & 28 & 26 & 0.75 \\
Total & 47 & 45 & 0.77 \\
\hline Abbrial
\end{tabular}

Abbreviations: LITA left internal thoracic artery, RA radial artery; SVG, saphenous vein graft

with 12 deaths in each group during the study period (log rank $=0.01, p=0.979$ ) (Fig. 1 ). There were 3 and 2 cardiac deaths in RA and SVG groups, respectively. There was no difference in long-term clinical outcome between the groups ( $\log$ rank $=0.450, p=0.509$ ) (Fig. 2). Eleven patients in RA group had one or more non-fatal events; 7 patients suffered a myocardial infarction, 9 patients underwent percutaneous coronary angioplasty, and 1 patient required redo coronary surgery. Likewise, 13 patients in SVG group had non-fatal event; 7 patients had myocardial infarction, 13 patients had percutaneous coronary intervention and 3 patients required redo coronary surgery.

\section{Repeated coronary angiography}

Repeated coronary angiography was performed in patients who had a positive physical load test or a new coronary event (unstable angina pectoris or myocardial infarction). In RA group 23 underwent repeated coronary angiography, whereas in SVG group 24 patients underwent this procedure. RA graft patency rate was $92 \%$, whereas SVG patency rate was $86 \%(p=0.67)$.

\section{Discussion}

This study reports on our series of 200 patients undergoing isolated, primary CABG using LITA grafting and the SVG in one group, and RA grafting as the second conduit in the second group. Our data indicate that there is no difference in the long-term clinical outcome between the patients in whom RA or SVG is used as a second conduit, beside LITA, for surgical myocardial revascularization. Additionally, graft patency in patients who underwent coronary angiography was similar between the groups.

\section{Clinical outcomes}

Two randomized clinical trials have reported that eventfree survival was greater in patients receiving a radial artery $[9,13]$. In the Stand-in-Y trial, event-free survival was similar in patients who received a radial artery 


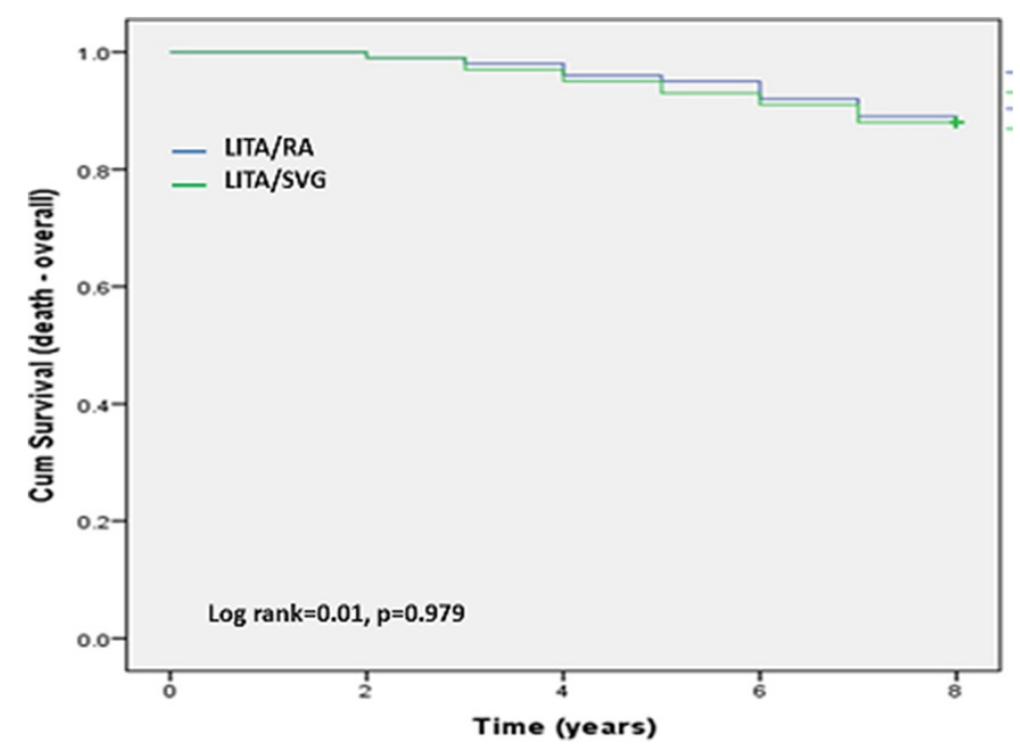

Fig. 1 Kaplan-Meier curve for total mortality. Abbreviations: LITA, left internal thoracic artery; RA, radial artery; SVG, saphenous vein graft

compared with a second ITA graft [13]. Two moderately large, single-center observational studies using propensity scores have recently been published $[14,15]$. Both early and late survival and event-free survival was enhanced with the use of a radial artery compared with a saphenous vein [15]. Perioperative outcomes including in hospital mortality $(0.1 \%$ for the RA patients and $0.2 \%$ for the SVG patients) were similar. Kaplan-Meier survival at 1,5 , and 10 years was $98.3,93.9$, and $83.1 \%$ for the RA group versus 97.2, 88.7, and $74.3 \%$ for the
SVG group (log rank, $p=0.0011$ ). Cox proportional hazards models showed a lower all-cause mortality in the RA group (hazard ratio 0.72 , confidence interval: 0.56 to $0.92, p=0.0084)$. Ten-year survivals showed a $52 \%$ increased mortality for the SVG patients (25.7\%) versus the RA patients $(16.9 \% ; p=0.0011)$. For symptomatic patients, RA patency was $80.7 \%$, which was not different than the LITA patency rate of $86.4 \%$ but was superior to the SVG patency rate of $46.7 \%(p<0.001)$. However, it appears that the use of RA yields inferior long-term

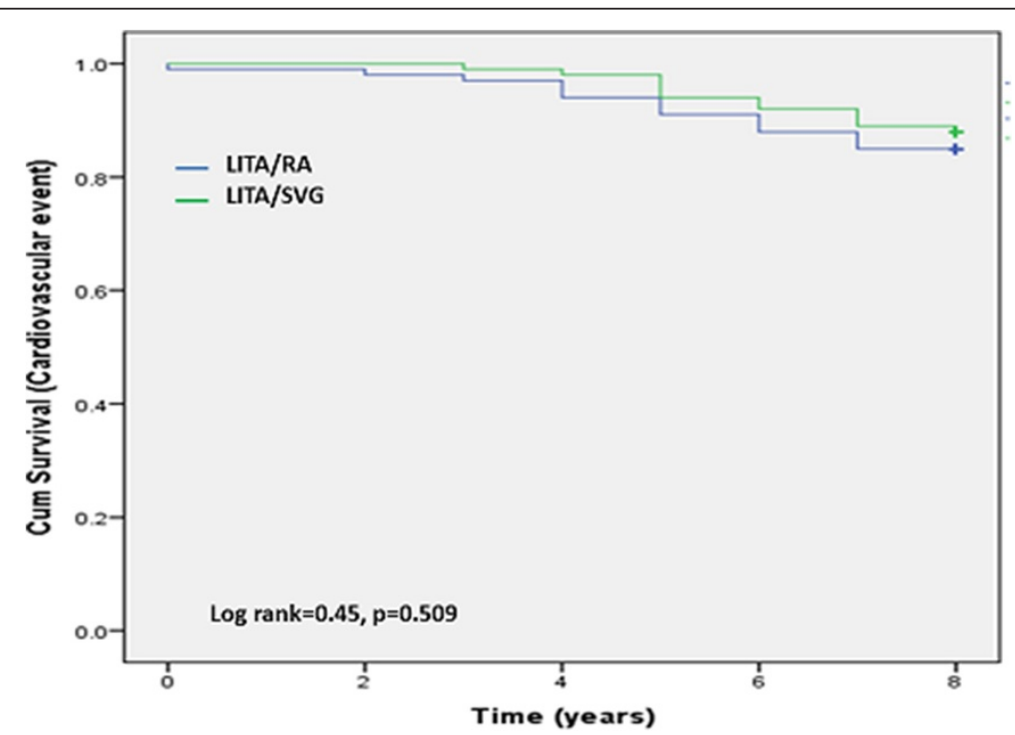

Fig. 2 Kaplan-Meier curve for long-term clinical outcomes (composite of cardiac death, non-fatal myocardial infarction and repeat revascularization). Abbreviations: LITA, left internal thoracic artery; RA, radial artery; SVG, saphenous vein graft 
clinical outcomes as compared to use of right internal thoracic artery as a second arterial conduit [16].

\section{Graft patency}

There are several reports of the medium to long-term clinical outcomes for RA grafting. Buxton and colleagues [11] in 2003 reported a prospective randomized study comparing the RA with the free RITA and the SVG. Their 5-year interim results did not support the hypothesis of superior patency of the RA compared with the RITA or the SVG. The most recent update from the same group in 2010 continued to show no differences in patency rates with pending clinical results [11].

Zacharias et al. [4] in 2004 evaluated the 6-year clinical outcomes of propensity-matched patients undergoing LITA-to-LAD grafting with either an additional RA graft or SVG as second conduit. In 925 propensity-matched patients, they found cumulative survival was better with the RA grafts. Angiographic data in restudied symptomatic patients showed a trend toward greater RA graft patency. They reported that the RA graft survival benefit remained when patients were subdivided on the basis of specific risk factors, with women, triple-vessel disease, younger patients (age $\leq 65$ years), and diabetic patients having a more pronounced survival benefit. However, the 11-year Kaplan-Meier analysis showed essentially identical RA versus SVG survival for the diabetic patients.

Desai et al. [17] in 2007 the Radial Artery Patency Study, examined randomized angiographic data in 440 RA versus 440 SVG grafts in CABG and showed RA was protective against occlusion, especially in women up to 12 months. A history of peripheral vascular disease was associated with higher risk of RA occlusion, while grafting to a vessel with proximal occlusion improved RA patency. The same group and others in 2008 showed, via angiographic data at 1-year post-CABG, that diabetes mellitus was an independent predictor of graft occlusion, although RA grafting was protective in this subgroup versus the SVG [17-21].

The RAPS study is the first multicenter clinical trial reporting radial graft patency beyond 5 years. In the other and larger multicenter CSP 474 VA trial [7], the radial artery or saphenous vein was allocated to the second-best target as determined by the surgeon; they reported that at 1 year, complete graft occlusion was similar in radial and study SVGs (11\%). a 5-year extension is underway. At 5.5 years, the single-center RSVP (Radial Artery Versus Saphenous Vein Patency) study from London, England, reported that complete graft occlusion was markedly less frequent in radial grafts compared with SVGs directed to the circumflex territory [8]. There was no apparent graft-by-territory interaction in the RAPS study, indicating that the relative benefit of the radial artery compared with the saphenous vein applies to both the right and circumflex territories. Graft patency was improved when the radial artery was directed to a more severely narrowed target vessel. The singlecenter Australian RAPCO (Radial Artery Patency and Clinical Outcomes) study scheduled angiographic followup within 5 years in a minority of patients and between 5 and 10 years post-operatively in the majority; they have published interim results from 5 to 10 years of follow-up [16].

Athanasiou et al. [14] included both randomized trials and observational studies in a meta-analysis to compare the patency rates across different follow-up intervals-there were 7 studies with a follow-up $>5$ years. We updated their review with results from the RAPS study and new data complete to April 2011 [22-25]. Radial grafting was associated with a reduced rate of late graft occlusion compared with saphenous veins (for observational and randomized trials, odds ratio: $0.520,95 \%$ confidence interval: 0.342 to $0.790, p=0.002$; and for randomized trials alone, odds ratio: $0.491,95 \%$ confidence interval: 0.314 to $0.766, p=0.002$.)

When the type of harvest of the RA is concerned, recent prospective, randomized, open-controlled trial that included 119 patients demonstrated that following 5 years of the initial operation both RA harvesting techniques (open and endoscopic harvest ) are safe, effective and result in excellent patency rates [26].

It is very difficult to develop an algorithm for the use of RA as a second conduit for surgical myocardial revascularization. Since it appears that RA is not superior in terms of clinical outcome to the vein grafts for the revascularization of the right coronary artery, we usually use RA for revascularization of the left side system. The main target for RA graft is reasonably sized $(\geq 1.5 \mathrm{~mm})$ obtuse marginal artery with at least $80 \%$ stenosis. However, decision about use of RA graft should be tailored individually in order to achieve greatest clinical benefit for the patient.

\section{Limitations of the study}

The major limitation of the trial is the relatively small number of patients. Additionally, the follow-up duration in our study group was relatively short (8 years), which may lead to the underestimation of net clinical benefit in patients in whom RA graft was used. However, since this was a single-center randomized trial and patients were followed for a considerable time, we believe that a meaningful conclusions may be drawn from our data.

\section{Conclusion}

In this small randomised study our data indicate that there is no difference in the 8 year clinical outcomes in relatively young patients between those having a RA or a saphenous vein graft used as a second conduit, beside LITA, for surgical myocardial revascularisation. 


\section{Competing interests}

The authors declare that they have no competing interests.

\section{Authors' contributions}

IP has made substantial contributions to conception and design, acquisition of data, analysis and interpretation of data; have been involved in drafting the manuscript and revising it critically for important intellectual content; have given final approval of the version to be published. DN has made substantial contributions to conception and design, analysis and interpretation of data; have been revising manuscript critically for important intellectual content; have given final approval of the version to be published. MP has made substantial contributions to conception and design, analysis and interpretation of data; have been revising manuscript critically for important intellectual content; have given final approval of the version to be published. PM has made substantial contributions to analysis and interpretation of data; have given final approval of the version to be published. OD has made substantial contributions to analysis and interpretation of data; have given final approval of the version to be published. DK has made substantial contributions to analysis and interpretation of data; have given final approval of the version to be published. NT has made substantial contributions to analysis and interpretation of data; have given final approval of the version to be published. BD has made substantial contributions to analysis and interpretation of data; have given final approval of the version to be published. PO has made substantial contributions to conception and design, analysis and interpretation of data; have been involved in drafting the manuscript and revising it critically for important intellectual content; have given final approval of the version to be published.

Received: 1 January 2015 Accepted: 5 October 2015

Published online: 15 October 2015

\section{References}

1. Loop F, Lytle B, Cosgrove D, Steward RW, Goormastic M, Williams GW, et al. Influence of the internal mammary-artery graft on 10-year survival and other cardiac events. N Engl J Med. 1986;314:1-6.

2. Carpentier A, Guermonprez $J$, Deloche A, Frechette C, DuBost C. The aortato-coronary radial artery bypass graft. A technique avoiding pathological changes in grafts. Ann Thorac Surg. 1973;2:111-21.

3. Slaughter MS. Conduits for surgical arterial revascularization: the answer is.... Circulation. 2011;124:1313-4.

4. Zacharias A, Schwann TA, Riordan CJ, Durham SJ, Shah AS, Habib RH. Late results of conventional versus all-arterial revascularization based on internal thoracic and radial artery grafting. Ann Thorac Surg. 2009;87:19-26.

5. Tranbaugh RF, Dimitrova KR, Friedmann P, Geller CM, Harris LJ, Stelzer P, et al. Coronary artery bypass grafting using the radial artery: clinical outcomes, patency, and need for reintervention. Circulation.

2012;126(11 Suppl 1):S170-5.

6. Dimitrova KM, Hoffman DM, Geller CM, Dienstag B, Cohen BC, Tranbaugh RF. Patency of endoscopically harvested radial artery grafts. Innovations. 2010;5:265-9.

7. Goldman S, Sethi GK, Holman W, Thai H, McFalls E, Ward HB, et al. Radial artery grafts vs saphenous vein grafts in coronary artery bypass surgery. JAMA. 2011:305:167-74.

8. Collins P, Webb CM, Chong CF, Moat NE. Radial artery versus saphenous vein patency randomized trial: five-year angiographic follow-up. Circulation. 2008;117:2859-64.

9. Desai ND, Cohen EA, Naylor CD, Fremes SE. A randomized comparison of radial-artery and saphenous-vein coronary bypass grafts. N Engl J Med. 2004;351:2302-9.

10. Tatoulis J, Buxton BF, Fuller JA, Meswani M, Theodore S, Power N, et al. Long-term patency of 1108 radial-coronary angiograms over 10 years. Ann Thorac Surg. 2009:88:23-30.

11. Buxton BF, Raman JS, Ruejgsakulrach P, Gordon I, Rosalion A, Bellomo R, et al. Radial artery patency and clinical outcomes: five year interim results of a randomized trial. J Thorac Cardiovasc Surg. 2003;125:1363-71.

12. Khot UN, Friedman DT, Pettersson G, Smedira NG, Li J, Ellis SG. Radial artery bypass grafts have an increased occurrence of angiographically severe stenosis and occlusion compared with left internal mammary arteries and saphenous vein grafts. Circulation. 2004;109:2086-91.
13. Nasso G, Coppola R, Bonifazi R, Piancone F, Bossetti G, Speziale G. Arterial revascularization in primary coronary artery bypass grafting: direct comparison of 4 strategies: results of the stand-in-Y mammary study. J Thorac Cardiovasc Surg. 2009;137:1093-100.

14. Athanasiou T, Saso S, Rao C. Radial artery versus saphenous vein conduits for coronary artery bypass surgery: forty years of competition-which conduit offers better patency? A systematic review and meta-analysis. Eur J Cardiothorac Surg. 2011;40:208-20.

15. Tranbaugh RF, Dimitrova KR, Friedmann P. Radial artery conduits improve long-term survival after coronary artery bypass grafting. Ann Thorac Surg. 2010;90:1165-72.

16. Possati G, Gaudino M, Prati F, Alessandrini F, Trani C, Glieca F, et al. Longterm results of the radial artery used for myocardial revascularisation. Circulation. 2003;108:1350-4.

17. Desai ND, Naylor CD, Kiss A, Cohen EA, Feder-Elituv R, Miwa S, et al. Impact of patient and target-vessel characteristics on arterial and venous bypass graft patency: insight from a randomized trial. Circulation. 2007;115:684-91.

18. Alexander JH, Emery Jr RW, Carrier M. MEND-CABG II Investigators Efficacy and safety of pyridoxal 5'-phosphate (MC-1) in high-risk patients undergoing coronary artery bypass graft surgery: the MEND-CABG ॥ randomized clinical trial. JAMA. 2008;299:1777-87.

19. Mentzer Jr RM, Bartels C, Bolli R. Sodium-hydrogen exchange inhibition by cariporide to reduce the risk of ischemic cardiac events in patients undergoing coronary artery bypass grafting: results of the EXPEDITION study. Ann Thorac Surg. 2008:85:1261-70.

20. Yau JM, Alexander JH, Hafley G. Impact of perioperative myocardial infarction on angiographic and clinical outcomes following coronary artery bypass grafting (from PRoject of Ex-vivo Vein graft ENgineering via Transfection [PREVENT] IV). Am J Cardiol. 2008;102:546-51.

21. Locker C, Schaff HV, Derani JA, Joyce LD, Park SJ, Burkhart M et al. Multiple arterial grafts improve late survival of patients undergoing coronary artery bypass graft surgery: analysis of 8622 patients with multivessel disease. Circulation. 2012;126:1023-30

22. Hayward PA, Gordon IR, Hare DL, Matalanis G, Horrigan ML, Rosalion A, et al. Comparable patencies of the radial artery and right internal thoracic artery or saphenous vein beyond 5 years: results from the Radial Artery Patency and Clinical Outcomes trial. J Thorac Cardiovasc Surg. 2010;139:60-7.

23. Ruttmann E, Fischler N, Sakic A, Chevtchik O, Alber H, Schistek R, et al. Second internal thoracic artery versus radial artery in coronary artery bypass grafting: a long-term, propensity score-matched follow-up study. Circulation. 2011:124:1321-9.

24. Achouh P, Isselmou KO, Boutekadjirt R, D'Alessandro C, Pagyny JY, Fouquet R, et al. Reappraisal of a 20-year experience with the radial artery as a conduit for coronary bypass grafting. Eur J Cardiothorac Surg. 2012;41:87-92.

25. Gaudino M, Possati G, Alessandrini F, Luciani N, Glieca F, Trani C, et al. Midterm clinical and angiographic results of radial artery grafts used for myocardial revascularization. J Thorac Cardiovasc Surg. 1998;116:1015-21.

26. Butns DJ, Swinamer SA, Fox SA, Romsa J, Vezina W, Akcinioglu C et al. Long term patency of endoscopically harvested radial arteries from a randomized controlled trial. Innovations. 2015;10:77-84.

\section{Submit your next manuscript to BioMed Central and take full advantage of:}

- Convenient online submission

- Thorough peer review

- No space constraints or color figure charges

- Immediate publication on acceptance

- Inclusion in PubMed, CAS, Scopus and Google Scholar

- Research which is freely available for redistribution 